\title{
DIFFERENTIAL HARNACK INEQUALITY FOR NONLINEAR HEAT EQUATIONS
}

\section{LIANG ZHAO}

Abstract. In this paper, we establish some differential Harnack inequalities for positive solutions to nonlinear heat equations coupled with the Bernhard List's flow. We also give some applications of these inequalities.

Mathematics subject classification (2010): Primary 53C44.

Keywords and phrases: Differential Harnack inequality, Bernhard List's flow, gradient estimate.

\section{REFERENCES}

[1] B. List, Evolution of an extended Ricci flow system, PhD Thesis. AEI Potsdam. http://www.diss.fu-berlin.de/2006/180/index.html (2006).

[2] X.-D. CAO, Z. Zhang, Differential Harnack estimates for parabolic equations, Complex and Differential Geometry, 8 (2011), 87-98.

[3] S. FAnG, Differential Harnack inequalities for heat equations with potentials under the Bernhard List's flow, Geom Dedicata, Doi 10.1007/s10711-011-9690-0.

[4] S.-Y. Hsu, Gradient estimates for a nonlinear parabolic equation under Ricci flow, (2008), arXiv: math.DG/0806.4004v1.

[5] L. MA, Hamilton type estimates for heat equations on manifolds, (2010), arXiv: math.DG/1009.0603v1.

[6] P. LI, S.-T. YAU, On the parabolic kernel of the Schrodinger operator, Acta Math., 156 (1986), 153-201.

[7] S.-T. YAU, On the Harnack inequalities of the partial differential equations, Comm. Anal. Geome., 2 (1994), 421-430.

[8] J.-Y. Wu, Differential Harnack inequalities for nonlinear heat equations with potentials under the Ricci flow, (2011), arxiv: math.DG/1009.1219V3.

[9] L. ZHAO, Differential Harnack inequality for the nonlinear heat equations, Proc. Japan Acad. Ser. A Math. Sci., 89 (2013), 96-99. 